\title{
11. Literaturhinweise und Weiterführendes zu Teil II
}

Der zweite Teil dieses Buches zeichnet sich dadurch aus, dass nur sehr wenig konkrete Physik betrieben wird, sondern vielmehr die Techniken und Fertigkeiten der Vektor- und Tensorrechnung vorgetragen und eingeübt werden. Dementsprechend besteht die (knappe) Liste der empfehlenswerten Bücher auch nicht aus Physiklehrbüchern, sondern aus Spezialabhandlungen für diese Themen. Die in diesem Teil hauptsächlich genutzte Literaturquelle ist das Buch von Bernard Schutz [35], in dem ähnlich wie hier die Vektor- und Tensorrechnung nicht nur über das Transformationsverhalten der Komponenten von Tensoren eingeführt wird, sondern die Tensoren selbst als Objekte (d.h. lineare Abbildungen) dargestellt und behandelt werden. Das ist - wenn man so will ein etwas ,modernerer" Ansatz als in vielen anderen Texten, die mit Koordinatentransformationen auskommen. Zu diesen gehört auch das Buch Vectors and Tensors von Daniel Fleisch [11], das einen leichten Einstieg in die Vektorund Tensorrechnung bietet.

$\mathrm{Zu}$ den weiterführenden Büchern im Bereich der Tensorrechnung gehören die Werke von Schutz Geometrical methods of mathematical physics [36] sowie von Jänich Vektoranalysis [20], die allerdings weit über das hinausgehen, was in diesem Teil betrachtet wurde und zudem eine abstrakte Darstellungsweise haben. 